\title{
Adjuvant Radiation Therapy for Distal Pancreatic Cancer: Is There a Role?
}

\author{
Brian G. Czito, MD and Christopher G. Willett, MD \\ Department of Radiation Oncology, Duke University Medical Center, Durham, NC
}

Pancreatic cancer is a major cause of cancer-related mortality worldwide. Little progress has been made in improving outcomes over the past 3 decades despite numerous trials encompassing many therapeutic agents and modalities. Except for rare anecdotes, pancreatic cancer is only curable when resection is undertaken. Unfortunately, more than $80 \%$ of patients are not resectable with either locally advanced/unresectable or metastatic disease at presentation. In the small percentage of patients who are resected, approximately $20 \%$ will survive 5 years, indicating that even "curative" therapy is ultimately palliative in nature. ${ }^{1}$ 5-fluorouracil (5-FU) stood as the backbone of systemic therapeutic regimens in this disease for many years until 1997 when gemcitabine was shown to result in a modest (median 1.3-month) survival benefit over 5-FU in patients with advanced disease. With a corresponding improvement in quality of life measures in patients receiving gemcitabine, this was considered the first major advance in this disease in more than a decade. ${ }^{2}$

Most patients with resectable disease will develop distant metastases and/or local recurrence following resection. Patterns of failure analyses from contemporary randomized trials have also demonstrated that resected patients, when treated with adjuvant chemotherapy only, experience unacceptably high rates of local recurrence. Therefore, it is reasonable to consider adjuvant therapies directed toward locally persistent disease, namely radiation therapy. In resectable patients, significant debate has arisen in recent years regarding the role of adjuvant radiation therapy, with widely diverging approaches. Both a small randomized trial as well as large institutional experiences from the United States have demonstrated a survival benefit in

(C) Society of Surgical Oncology 2010

Published Online: 29 September 2010

B. G. Czito, MD

e-mail: czito001@mc.duke.edu patients receiving adjuvant radiation therapy following pancreatic cancer resection. ${ }^{3,4}$ In contrast, a study from the EORTC did not confirm a significant survival benefit from adjuvant radiation therapy and chemotherapy. ${ }^{5}$ In addition, a large study from the ESPAC group (ESPAC-1) did not confirm radiation therapy benefit and in fact suggested a detriment to its use, reporting that any survival benefit from adjuvant therapy comes from systemic therapy with 5-FU. ${ }^{6}$ This trial has been appropriately criticized for significant methodologic weaknesses, while still confirming that local recurrence remains a dominant form of failure in patients treated in this study. In a follow-up study from the ESPAC group (and in contrast to previously reported findings in advanced disease), preliminary results of the ESPAC-3 study showed that adjuvant gemcitabine did not confer any survival advantage when compared with the historical standard 5-FU. ${ }^{7}$ A phase III study from the RTOG (9704) compared adjuvant gemcitabine delivered before and following 5-FU based chemoradiotherapy with a similar regimen using 5-FU before and following the same chemoradiotherapy regimen. ${ }^{8}$ This study contained a greater number of distal pancreatic cancer patients versus other trials and analyzed outcomes between proximal and distal lesions. While the original report described a survival benefit in pancreatic head patients receiving adjuvant gemcitabine-based therapy, no survival advantage was seen with gemcitabine use in patients with body and tail lesions.

While these randomized trials have included and focused on outcomes in patients with pancreatic head adenocarcinoma, a minority of patients $(15-20 \%)$ will present with pancreatic body or tail lesions. Because these tumors are less common, little data exist describing outcomes and patterns of failure compared with patients with pancreatic head lesions. It is also unclear whether there are distinct biological differences between proximal and distal pancreatic tumors. Historically, patients with distal tumors have a worse prognosis compared with pancreatic head 
lesions, primarily because of a lack of symptoms, precluding early diagnosis and resulting in advanced stage at presentation in many cases. As in patients with pancreatic head lesions, even in the minority that present with resectable (and therefore potentially curable) distal disease, prognosis remains poor. Given that distal pancreatic cancers compose a small minority of patients with this malignancy, adjuvant treatment recommendations are usually extrapolated from studies of pancreatic head malignancies, where outcomes also remain poor, optimal adjuvant regimens uncertain, and existing adjuvant strategies modestly effective at best.

To date, limited data examining the role of adjuvant or neoadjuvant therapy for distal pancreatic cancers have been reported. In the current issue of Annals of Surgical Oncology, Redmond et al. describe the outcomes of 94 evaluable patients undergoing distal pancreatectomy for adenocarcinoma of the pancreatic neck, body, and tail at Johns Hopkins University, most of whom received adjuvant fluoropyrimidine-based chemoradiotherapy. ${ }^{9}$ Outcome comparison of adjuvantly irradiated patients was made to similar patients undergoing surgery alone over the same time period. In summary, no significant overall survival difference was seen between patients treated adjuvantly versus those with surgery alone. Although median survival was higher in the adjuvantly treated group, this did not reach statistical significance (16.7 vs. 12.1 months, $P=.23$ ). On subset analysis, a possible survival benefit in patients with lymph node metastases receiving adjuvant chemoradiotherapy was suggested (estimated 5-year survival 15 vs. $0 \%$ ).

Limitations of this study include its retrospective analysis with selection biases, small sample size, variability in systemic agents, a high percentage of treatment breaks in irradiated patients, and variability of radiation technique. Nonetheless, it is a unique (and to our knowledge the largest) study assessing the role of adjuvant therapy in this patient subgroup and is an important contribution to the literature of this malignancy. A caveat of this study is that most irradiated patients were treated outside of Johns Hopkins, with details of therapy unable to be fully ascertained. Whether this influenced patient outcomes in this series, however, is unknown. It is important to note that pancreatic cancer radiation field design deviation was shown to result in inferior outcomes in the RTOG 97-04 study. ${ }^{10}$ Similarly, the issue of field design and quality assurance is relevant to distal lesions given that patterns of locoregional spread differ significantly relative to pancreatic head lesions, mandating correspondingly different radiation fields relative to pancreatic head tumors. ${ }^{6,11}$ Along these lines, a subgroup analysis of evaluating patients irradiated at Johns Hopkins versus outside institutions revealed a significant survival difference in favor of patients treated at the former (median survival 21.5 vs.
14.5 months, $P<.01$ ), although this was only of borderline significance when adjusting for other variables. Similarly, when analysis was limited to patients treated at Johns Hopkins versus those undergoing observation alone, a significant survival difference was again seen (21.5 vs. 12.1 months, $P=.04$ ), although this was again of borderline significance when adjusting for other variables. Also noteworthy is that nearly $20 \%$ of patients under evaluation for adjuvant therapy following pancreatectomy were excluded because of the development of metastatic disease shortly after surgery, consistent with what has been observed in pancreatic head patients considered for adjuvant therapy. This observation suggests further investigation of neoadjuvant approaches in this disease are indicated in efforts to avoid the morbidity of a noncurative surgery in patients with subclinical metastases displaying rapid progression, as well as avoidance of radiation-related bowel toxicity that may be increased following distal pancreatectomy.

Clearly, the waters of pancreatic cancer adjuvant therapy remain muddy and debate surrounds the role of radiation therapy in this setting. The newly opened, internationally run RTOG 0848/EORTC study will seek to clarify the role of adjuvant radiation therapy, following delivery of full-course gemcitabine-based chemotherapy. Importantly, this trial mandates an array of quality assurance measures in surgery, pathology, and chemotherapy as well as radiation therapy. However, patients with distal pancreatic lesions are excluded from eligibility, and it is likely that adjuvant treatment recommendations in distal lesions will continue to be based on extrapolations from studies evaluating proximal lesions. Despite this, with currently used therapeutic regimens, any gains in this disease will likely be incremental at best, making investigation of newer and novel treatment paradigms mandatory.

\section{REFERENCES}

1. Geer R, Brennan M. Prognostic indicators for survival after resection of pancreatic adenocarcinoma. Am J Surg. 1993; 165:68-72; discussion 72-3.

2. Burris HA 3rd, Moore MJ, Andersen J, Green MR, Rothenberg, ML, Modiano MR, et al. Improvements in survival and clinical benefit with gemcitabine as first-line therapy for patients with advanced pancreas cancer: a randomized trial. J Clin Oncol. 1997; 15:2403-13.

3. Kalser M, Ellenberg S. Pancreatic cancer. Adjuvant combined radiation and chemotherapy following curative resection. Arch Surg. 1985;120:899-903.

4. Herman J, Swartz M, Hsu C, Winter J, Pawlik TM, Sugar E, et al. Analysis of fluorouracil based adjuvant chemotherapy and radiation after pancreaticoduodenectomy for ductal adenocarcinoma of the pancreas: results of a large, prospectively collected database at the Johns Hopkins Hospital. J Clin Oncol. 2008;26: 3503-10. 
5. Klinkenbijl J, Jeekel J, Sahmoud T, van Pel R, Couvreur ML, Veenhof $\mathrm{CH}$, et al. Adjuvant radiotherapy and 5-fluorouracil after curative resection of cancer of the pancreas and periampullary region: phase III trial of the EORTC gastrointestinal tract cancer cooperative group. Ann Surg. 1999;230:776-82; discussion $782-4$.

6. Neoptolemos J, Stocken D, Friess H, Bassi C, Dunn JA, Hickey $\mathrm{H}$, et al. A randomized trial of chemoradiotherapy and chemotherapy after resection of pancreatic cancer. $N$ Engl J Med. 2004;350:1200-10.

7. Neoptolemos J, Büchler M, Stocken DD, Ghaneh, P, Smith D, Bassi C, et al. ESPAC-3(v2): A multicenter, international, openlabel, randomized, controlled phase III trial of adjuvant 5-fluorouracil/folinic acid (5-FU/FA) versus gemcitabine (GEM) in patients with resected pancreatic ductal adenocarcinoma. $J$ Clin Oncol. 2009;27:18s (suppl; abstr LBA4505).
8. Regine W, Winter K, Abrams R, Safran H, Hoffman JP, Konski A, et al. Fluorouracil vs. gemcitabine chemotherapy before and after fluorouracil-based chemoradiation following resection of pancreatic adenocarcinoma: a randomized controlled trial. JAMA. 2008;299:1019-26.

9. Redmond KJ, Wolfgang CL, Sugar E, Ahn J, Nathan H, Laheru $\mathrm{D}$, et al. Adjuvant chemoradiation therapy for adenocarcinoma of the distal pancreas. Ann Surg Oncol. 2010. doi:10.1245/s10434010-1200-3 [Epub ahead of print].

10. Abrams R, Winter K, Regine W, Safran H, Hoffman JP, Konski AA, et al. Correlation of RTOG 9704 (adjuvant therapy of pancreatic adenocarcinoma) radiation therapy quality assurance scores with survival. J Clin Oncol. 2007;25:18s, 4523.

11. Sun W, Leong C, Zhang Z, Lu JJ. Purposing the lymphatic target volume for elected radiation therapy for pancreatic cancer: a pooled analysis of clinical evidence. Radiat Oncol. 2010;5:28. 